\title{
Taxonomy of Induction-Motor Mechanical-Fault Based on Time-Domain Vibration Signals by Multiclass SVM Classifiers
}

\author{
Purushottam Gangsar $^{1}$ - Rajiv Tiwari ${ }^{1}$
}

Received: 10 July 2016 / Revised: 18 August 2016 / Accepted: 19 August 2016 / Published online: 3 October 2016

(C) Springer Science+Business Media Singapore 2016

\begin{abstract}
In the present work, faults in induction motors (IM) have been diagnosed by multiclass support vector machine (SVM) algorithms based on time domain vibration signals. The main focus is to classify mechanical faults of induction motors, i.e. the bearing fault, unbalanced rotor, bowed rotor and rotor misalignment at different rotational speeds and diverse loading conditions. In this work, an induction motor test setup was used to generate vibration signals of seeded mechanical faults. For the effective fault diagnosis, one-versus-one multiclass SVM approach with the Gaussianradial basis function (RBF) kernel has been used. For the fault classification, firstly optimum statistical features from higher statistical moments have been selected. Also the selection of SVM kernel parameters, numbers of feature datasets and optimum ratio of training-to-testing data have been performed. The SVM classifier is trained and tested at the same rotational speeds as the measured data as well as innovatively tested at intermediate rotational speeds for which measured data was not available. It is observed that classification accuracy gradually increases with the increase of the rotational speed and with the increase of the load on the IM.
\end{abstract}

Keywords Induction motor (IM) - Mechanical faults . Multi-fault classification - Support vector machine (SVM) . Radial basis kernel (RBF) · One-versus-one (OVO) approach

Rajiv Tiwari

rtiwari@iitg.ernet.in

1 Department of Mechanical Engineering, Indian Institute of Technology Guwahati, Guwahati, Assam 781 039, India

\section{Introduction}

Induction motors or squirrel-cage motors are nowadays commonly used prime movers in a wide field of light and heavy duty rotating machine applications in all types of industry. This is due to their simple construction, handiness, reliability, low maintenance requirements, low cost, adaptability to wide variety of operation conditions and availability of power converters using adequate control approach. However, induction motors are prone to many types of faults in industry that causes a complete motor failure, which in turn may cause huge production losses in terms of the production cost, production time, wastage of raw material and also chances of human injuries. Induction motors usually falls out of service not just due to the age or operating hours but due to the unusual mechanical load, power supply deviation, improper or inadequate lubrications, more heat and ineffectual sealing. Hence, the early detection of faults in induction motors is very important in order to prevent the complete failure of motor and unexpected huge production losses in industry [1].

In recent years, many condition based monitoring (CBM) techniques have been employed for early detection of faults in IM, i.e. machine current signature analysis (MCSA), vibration, air gap torque, acoustic noise measurement, instantaneous angular speed and temperature measurement [2]. In order to find the most effective CBM techniques for the detection of various faults in IM, Timusk et al. [3] compared the vibration CBM with the MCSA for the detection of broken rotor bar (BRB) and bearing faults for different load conditions and speeds, and finally concluded that the vibration based CBM was the most effective technique for the bearing fault detection while the MCSA was the best for the BRB detection. Kral et al. [4] suggested that the vibration based CBM technique was reliable technique for the detection of bearing and other mechanical faults in the IM. Many 
researchers have been suggested to use vibration signal for condition monitoring and fault diagnosis of mechanical as well as electrical faults in IM, due to its easy measurability, high accuracy and effectiveness in signal analysis that represent the actual machine condition, among others. Condition monitoring of machines can be performed through signal analysis in time, frequency and time-frequency domain [5].

Various artificial intelligence techniques have been successfully used for the fault taxonomy in IM, such as artificial neural network (ANN), fuzzy logic, principal component analysis (PCA) and linear discriminant analysis (LDA), classification and regression tree (CART), and immune genetic system $[6,7]$. A relatively new AI method, SVM is also used as an artificial intelligence technique for the fault detection of IM $[5,8]$. In order to compare these AI methods for fault diagnosis, Samanta [9] performed the gear fault detection using ANN and SVM with genetic algorithm, and concluded that the SVM can perform well in comparison with ANN even with smaller number of samples and also training time is less in case of the SVM. Silva and Pederiva [10] performed the induction motor fault detection using artificial intelligence techniques, like SVM, fuzzy logic and ANN, and concluded that the SVM has a good generalization, among others.

Nowadays, SVM has gained popularity for fault diagnosis over other methods, due to its generalization capabilities and computational cost. The SVM has been extended to multiclass-classification from binary classification and also various kernels have been developed to handle nonlinear problem through the SVM. In order to compare multiclass SVM techniques, Hsu and Lin [11] and Hsu et al. [12] presented a comparison among different methods of multiclass SVM through different kernels, and concluded that oneversus-one method is most effective for the classification, among one-versus-one (OVO), one-versus-all (OVA) and direct-acyclic graph SVM (DAGS). RBF kernels produced better result among other kernel such as, linear, polynomial and sigmoid.

For effective fault classification through the SVM, data preparation, i.e. feature extraction and selection, is a critical step. Feature extraction methods such as principal component analysis (PCA) and independent component analysis (ICA) and feature selection techniques such as the genetic algorithm (GA) and decision tree have been introduced in the literature. In order to evaluate the work related to fault diagnosis using SVM, Tiwari and Bordoloi [13] performed multi-fault classification of gears based on the SVM. Genetic algorithm, grid-search method and artificial bee colony algorithm were used for optimizing SVM parameters. Li et al. [14] performed fault diagnosis of rolling element bearings by the SVM. Improved ant colony optimization (IACO) algorithm was used for optimization of the SVM parameter. Baccarini et al. [8] presented a practical industrial application of the SVM for mechanical faults diagnostic of IMs based on frequency-domain signals. However, bearing faults which is one of critical faults in IM were not included. Nguyen and Lee [5] presented a technique for diagnosing mechanical faults in IMs based on the vibration by using the SVM. The genetic algorithm (GA) was used to select the useful statistical features and SVM parameters. However the study was mainly focused on selection of useful features and parameters. Widodo et al. [15] presented a combination of the independent component analysis (ICA), the principal component analysis (PCA) and the SVM for the fault diagnosis of IMs based on the vibration signature and MCSA. They showed that the combination of ICA and SVM could serve as an encouraging alternative and also demonstrated the application of non-linear feature extraction and SVMs could serve as an alternative. Morales et al. [16] introduced the data fusion by using the multi-class SVM to detect mechanical faults in IMs using the vibration and line-current signatures.

Chattopadhyay and Konar [17] presented feature extractions using wavelet transforms, i.e. continuous wavelet transform (CWT) and discrete wavelet transform (DWT), and feature selections using the greedy-search technique for the multi-class fault detection of IM using the RBF neural networks, multilayer perception (MLP) neural networks and SVM classifiers. Uddin et al. [18] and Shahriar et al. [19] presented the fault diagnosis of IMs using the texture feature extraction and the multiclass SVM. In this work, they converted time-domain vibration signals to 2-D gray images by transforming the amplitude of the signal into the intensity of pixels in an image. However, robustness of texture feature extraction was not justified in high noisy industrial environment; also it had high computational cost. From literatures, it is apparent that very few researches have been worked for the fault diagnosis of IM, especially of all possible mechanical faults together, by using multi-class SVM algorithms based on vibration signatures. For the fault diagnosis of induction motor, hardly any researcher has examined the SVM performance at various operating conditions of induction motor, i.e. a wide range of speed as well as torque load. For cases when vibration data is available at discrete speeds hardly any attempt has been made for the fault classification at intermediate speeds. Moreover, the holistic optimal selection of diverse SVM parameters has been hardly attempted. Looking at the reported ability of SVM attempting these issues would be a real beneficial and useful for industries towards automation of condition based monitoring systems.

The present work deals with the mechanical multi-fault classification of induction motors based on time domain vibration signals by using multiclass SVM algorithms. Firstly, parametric studies have been performed for the selection of optimum statistical features, the optimum Gaussian RBF kernel parameter (gamma), training-testing data ratio and the number of feature datasets. After selecting these parameters, the SVM capability to classify mechanical faults 
in IMs has been presented based on the one-versus-one approach. The aim of this research is to examine the SVM fault prediction capability for various operating conditions of IMs, i.e. a wide range of rotational speeds and different loadings on the IM. Firstly, the SVM capability has been examined when classifier is tested at same rotational speed as training for all operating conditions. Then after modifying the SVM algorithm, again the SVM capability has been examined when the classifier innovatively tested at an intermediate rotational speed for which classifier was not trained.

\section{Support Vector Machine Algorithms}

SVMs are popular learning machines based on statistical learning theory that analyze data and recognizes patterns. The basic SVM is based on the binary classification but recently it has been extended to the multiclass classification for handling the real world situation. The binary and multiclass SVMs are described below in brief.

\section{Binary SVMs}

The base of SVM has been developed by Vapnik (1995) and is earning popularity due to many provocative features and encouraging empirical performances. The basic SVM is based on the binary classification, which construct a hyperplane or a set of hyperplanes in a high or infinite dimensional space, which could be used for the further classification. Figure 1 depicts the optimal separating hyper-planes, it is called good separation if the hyper-planes have a largest distance to the closest training data point of any class. Figure 2 shows the SVM classification problem solution by maximizing the margin between two classes. Hence, for a higher margin, the generalization error will be very less. However, if a set of training data are fed to the SVM each specified

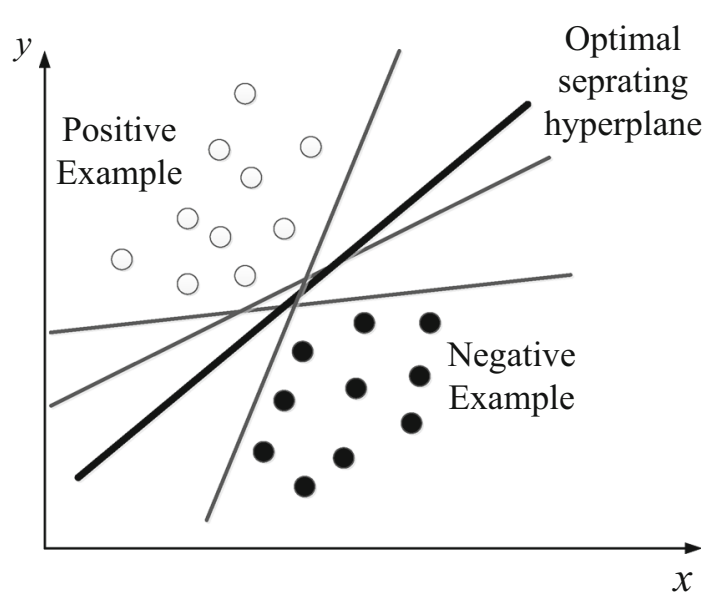

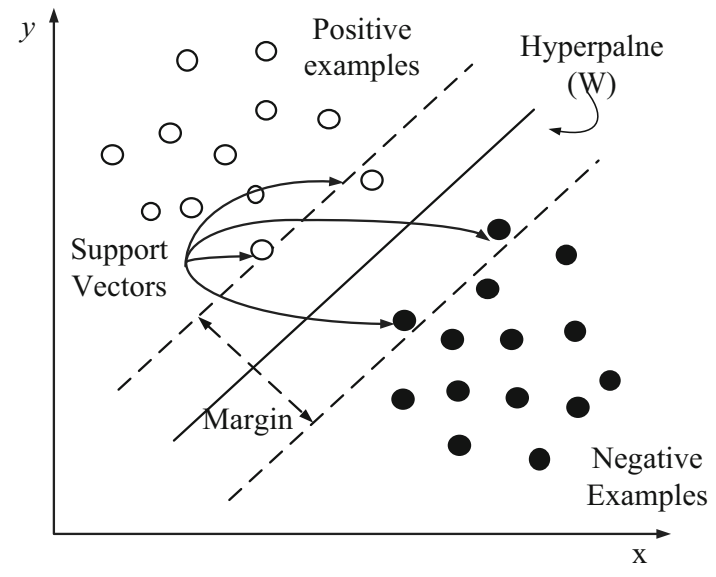

Fig. 2 The separation of positive and negative examples by an optimal separation hyperplane

as one of two different classes, the SVM algorithm builds a classification model that predicts if the new data falls into one class or to other class [20,21].

The SVM is based on the principle of structural risk minimization (SRM), which has been proved to be superior to the empirical risk minimization (ERM), used by the ANN. The ERM minimizes the error on training sets while the SRM minimizes an upper bound on the expected risk. And that is why the SVM has better generalization ability than others, which is the main motive of the statistical learning. And also the kernel function has been introduced with the SVM to deal with the non-linear classification problem [22,23].

For an optimal separating hyperplane, consider a problem to separate the sets of training vectors of two different classes,

$$
\begin{gathered}
p=\left\{\left(x_{1}, y_{1}\right),\left(x_{2}, y_{2}\right), \ldots,\left(x_{n}, y_{n}\right)\right\}, \\
x \in R^{m}, \quad y \in(-1,1)
\end{gathered}
$$

with a hyperplane,

$(w, x)+b=0$

Hence, the hyperplane that optimally separates the data, is one that minimizes the following function

$\operatorname{Min}\{\tau(w, b)\}=\frac{1}{2} w^{2} \quad$ Subject to,$y_{i}\left\{w^{T} \phi\left(x_{i}\right)+b\right\} \geq 1$

This problem is very difficult to solve directly because the constraints are quite complex. Hence, Lagrangian duality theory is used for simplifying this problem. This approach leads to solving the following dual problem

$\operatorname{Max}\{D(\alpha)\}=\sum_{i=1}^{m} \alpha_{i}-\frac{1}{2} \sum_{i, j=1}^{m}\left\{y_{i} \alpha_{i} y_{j} \alpha_{j} \phi\left(x_{i}\right)^{T} \phi\left(x_{j}\right)\right\}$

Fig. 1 Optimal separating hyperplane 
with,

$\sum_{i=1}^{m} y_{i} \alpha_{i}=0$ and $\alpha_{i} \geq 0, i=1,2, \ldots, m$.

This dual Problem is computationally easier because its constraints are much simpler. The solution $\alpha^{*}$ of the dual optimization problem [i.e., Eq. (5)] recovers the direction $w^{*}$ of the optimal hyperplane.

$w^{*}=\sum_{i=1}^{m} \alpha_{i}^{*} y_{i} \phi\left(x_{i}\right)$

It becomes simple one dimensional problem for determining the bias $b^{*}$. The linear discriminate function can then be written as

$\hat{y}(x)=w^{* T} x+b^{*}=\sum_{i=1}^{m}\left\{y_{i} \alpha_{i}^{*} \phi\left(x_{i}\right)^{T} \phi(x)\right\}+b^{*}$

This above problem can solve a linear case but in other cases where the linear boundary is not enough to separate two different classes in input spaces properly, i.e. the nonlinear classification, then the SVM mapping is required to map the $N$-dimensional input vector into a higher dimensional feature space. The linear optimization problem and the linear discriminant function only associate patterns $x$ through the dot products computation in the feature space. And it can compute the dot product directly then there is no need to compute the features function, $\phi(x)$. Rather than choosing, $\phi(x)$, Boser et al. [24] introduced to directly select a kernel function $k\left(x, x^{\prime}\right)$ that substitute a dot product $\phi(x)^{T} \phi\left(x^{\prime}\right)$ in some undefined high dimensional space. Then the basic form of SVM will be

$\hat{y}(x)=\sum_{i=1}^{m}\left\{y_{i} \alpha_{i} K\left(x, x_{i}\right)\right\}+b$

The above formulation of SVM is based on the binary classification, and it can handle the multiclass case by simply combining it. In next subsection, the multiclass SVM method will be discussed briefly.

\section{Multiclass SVM}

The basic SVM based on the binary classification deals with only two classes, i.e. the positive and negative classes but in reality more than two class are found. In present, there are two types of approaches available for solving the multiclass SVM, the first constitutes and combines many binary classifiers, however; the second directly considers all data in a single larger optimization problem. It is computationally less expensive to solve a binary classification problem than a multiclass problem with same numbers of data. Hence, for handling such multiclass problem, methods like one-versusone (OVO), one-versus-all (OVA) and direct-acyclic graph SVM (DAGS) have been introduced. These methods disintegrate the multiclass problem into several binary classes [11]. The OVA approach constitutes $k$-SVM models, where $k$ is the number of classes, the OVO approach constitutes $k(k-1) / 2$-SVM models and each one trains data from two different classes. Whereas, by the DAGS approach, the training method is same as the OVO by constituting $k(k-1) / 2$ SVM models but in testing it uses a rooted binary DAG, which has $k(k-1) / 2$ internal nodes and $k$ leaves. Hsu and Lin [12] concluded that OVO is an effective method for practical use among others. In this work, LIBSVM [25] software has been used to the multiclass fault classification of IM using the OVO approach.

\section{Experimental Setup and Experimentation}

In the present study, the actual set-up for the IM fault diagnosis consists of the Machine Fault Simulator ${ }^{\mathrm{TM}}$ (MFS), tri-axial accelerometer, constant DC power source, data acquisition system (National Instrument ${ }^{\mathrm{TM}}$ ) and a signal monitor as shown in Fig. 3. However, the MFS was used to perform the experiment that consisted of a 3- $\phi$ induction motor (4-pole, 1.6 kVA, 4.2 A, 0-240 V output voltage, $1-400 \mathrm{~Hz}$ frequency range, max RPM-10,000, with lubricated ball bearing, controller-Delta VFD-S, number of rotor bars-34, number of stator slots-24), shaft, coupling, pulley with V-type belt, gear box assembly (single stage bevel gear), permanent magnet clutches and brakes (torque controller) and one photovoltaic sensor with reflecting tape on the shaft as shown in Fig. 4. The tri-axial accelerometer (sensitivity: $100.3 \mathrm{mV} / \mathrm{g}-x$ axis, $100.7 \mathrm{mV} / \mathrm{g}-y$ axis, $101.4 \mathrm{mV} / \mathrm{g}$ $-z$ axis) was mounted on the top of IM at the end opposite to cooling fan, for measuring the linear acceleration in all three

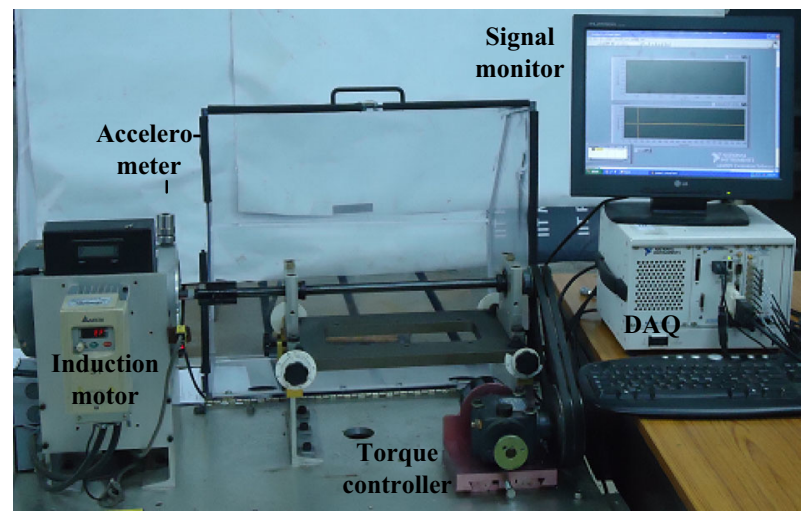

Fig. 3 Experiment setup with MFS 


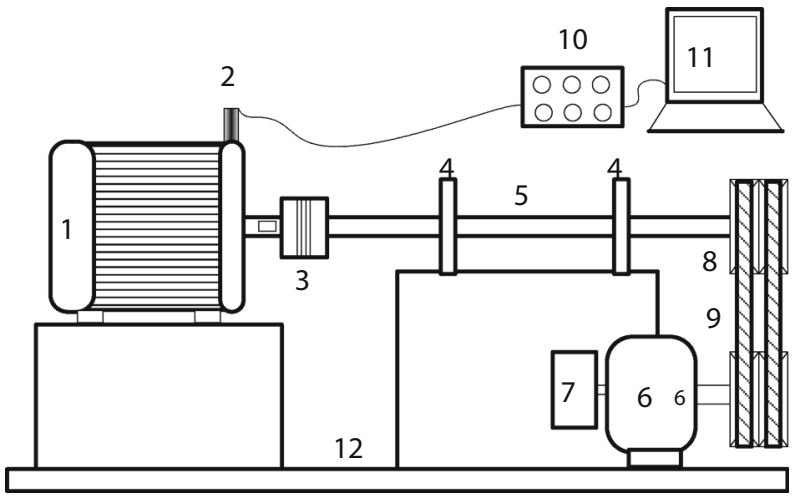

Fig. 4 A schematic line diagram of the experiment setup (1 Test IM, 2 Tri-axial accelerometer, 3 Coupling, 4 Bearings, 5 Shaft, 6 Gear box, 7 Torque controller, 8 Pulley, 9 Belt, 10 DAQ, 11 Signal monitor and 12 base plate)

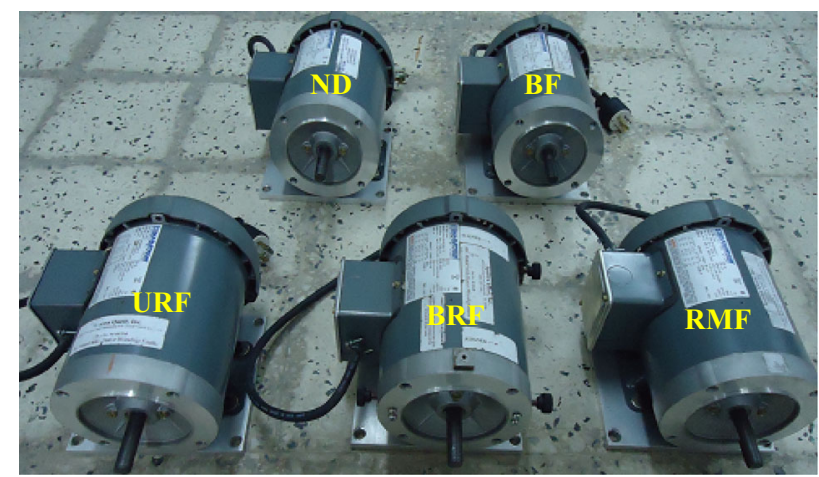

Fig. 5 Five Induction motors with individual faulty conditions ( $N D$ no defect, BF: bearing fault, $U R F$ unbalance rotor fault, $B R F$ bowed rotor fault and $R M F$ rotor misalignment fault)

orthogonal directions in time domain. A permanent magnet clutches and brakes (range from 0 to $0.565 \mathrm{~N}-\mathrm{m}$ ) were used to vary the load on IM. The photovoltaic sensor, which requires DC power source, was mounted near the coupling to measure the rotational shaft speed. In the present study, five types of faulty IM namely (1) the Bearing Fault (BF), (2) the Rotor Misalignment Fault (RMF), (3) the Bowed Rotor Fault (BRF), (4) the Unbalanced Rotor Fault (URF) and (5) a Healthy Motor (HM or no defect) were considered as shown in Fig. 5.

Vibration measurements were monitored on a Bruel and $\mathrm{Kjaer}^{\mathrm{TM}}$ FFT analyzer and measurement simulation were processed on Pulse ${ }^{\mathrm{TM}}$ Lab Shop of version 7.0.1.298. Datasets were collected for the range of $10-40 \mathrm{~Hz}$ in the interval of $5 \mathrm{~Hz}$ for each fault condition. For a dataset, 2000 samples were collected in time domain at the rate of 20,000 samples/s. In the similar manner, total of 300 datasets were collected for linear vibrations (in $x$-horizontal, $y$-axial, and $z$ vertical directions). Also measurements were taken for three different load conditions $(0,0.113$ and $0.565 \mathrm{~N}-\mathrm{m})$ from the no-torque to the high-torque applied to the IM.

\section{Extraction of Statistical Features}

In the present study, total eight useful statistical features were generated using higher statistical moments from the raw vibration signal (one axial and two radial directions of the motor) in time domain for the effective fault identification of the IM. In this work, the probability density function, $p(x)$, has been used for the vibration waveform to extract the features. i.e., the total probability is the $0^{\text {th }}$ moment (i.e., one), the mean is the first moment, the variance (square of standard deviation) the second moment, the skewness is the third moment, the kurtosis is the fourth moment, the hyper-skewness is the fifth moment and the hyper-flatness is the sixth moment of statistics.

For the vibration data, consider the mean $\mu_{1}=\bar{x}_{i}$ and the variance $\sigma^{2}$ ( $\sigma$ - the standard deviation), $k^{\text {th }}$ moments of about mean $\mu_{k}[26,27]$, as

$$
\begin{aligned}
& \mu_{1}=\bar{x}=\frac{1}{N} \sum_{i=1}^{N} x_{i}^{k} \\
& \mu_{2}=\sigma^{2}=\frac{1}{N} \sum_{i=1}^{N}\left(x_{i}-\mu_{1}\right)^{2} \\
& \mu_{k}=\frac{1}{N} \sum_{i=1}^{N}\left(x_{i}-\mu_{1}\right)^{k}
\end{aligned}
$$

Higher statistical moments up to $k=8$ have been used as statistical features for the present study, which are calculated from 2000 data points in a dataset. Higher statistical moments of acquired signals for BRF in $x$-direction at $40 \mathrm{~Hz}$ rotating speed are shown in Fig. 6. Total 300 datasets were collected from test rigs for three direction linear vibrations, so total $3 \times 8 \times 300$ feature points were available for each of IM fault cases.

\section{Experimental Results and Discussions}

The multi-fault classification of IM faults (mainly mechanical fault conditions like the BF, URF, BRF, RMF and ND) have been considered based on the multiclass SVM. Hence, the study consists of the data generation and acquisition, signal processing, feature extraction, selection of effective features, selection of RBF kernel parameter, selection of the optimum ratio of training and testing data, selection of optimum number of datasets and finally the fault diagnosis using the multiclass SVM.

For the effective performance of fault classification using the SVM, first feature points were divided into the training data and the final testing data for the SVM in various 

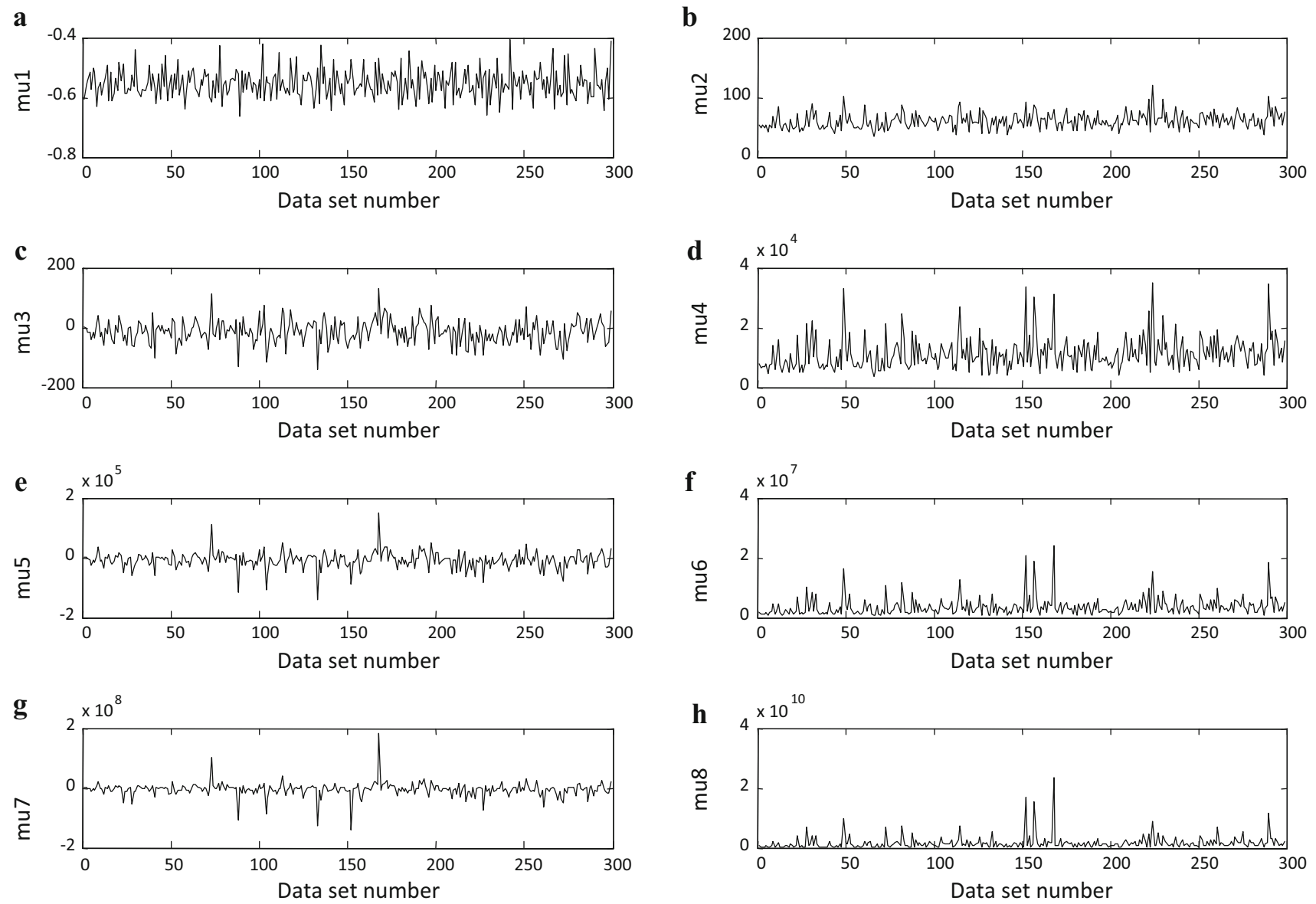

Fig. 6 Typical higher statistical features of acquired signals for the BRF at $40 \mathrm{~Hz}$ rotating speed (in $x$-direction)

ratios. Then one-versus-one multiclass SVM approach and Gaussian-RBF kernel (which is one of the very popular and claimed to be very effective kernel) were used to classify the different faulty conditions effectively in all exercises of selection of best parameters and also in final fault diagnosis. The training of SVM was performed at particular discrete rotational speeds followed by the testing of the SVM at the same rotational speeds as the training. Then the testing is also performed at the intermediate rotational speeds that of measurements. And finally results of fault diagnosis are obtained in the form of generalization accuracy, i.e. prediction levels of the testing data as

\section{Generalization accuracy}

$$
=\left(\frac{\text { number of correctly predicted data }}{\text { total number of testing data }}\right) \times 100
$$

Figure 7 shows the flowchart, which summarizes the fault diagnosis of IM using the SVM technique.

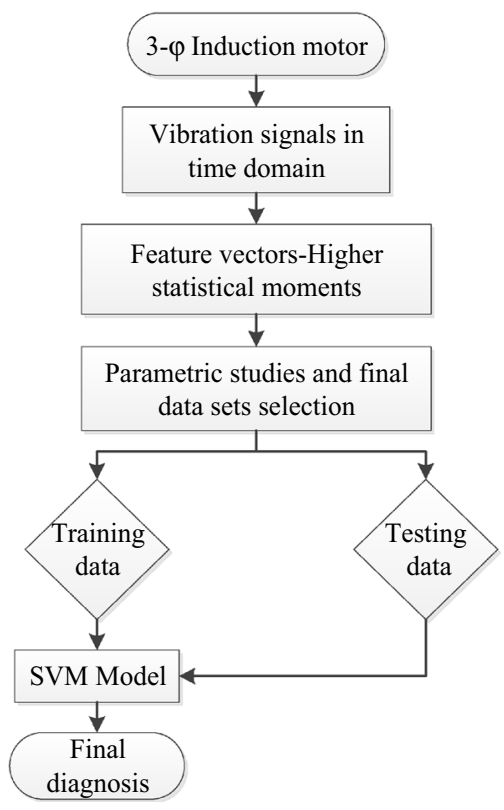

Fig. 7 A flow chart of the IM fault diagnosis 


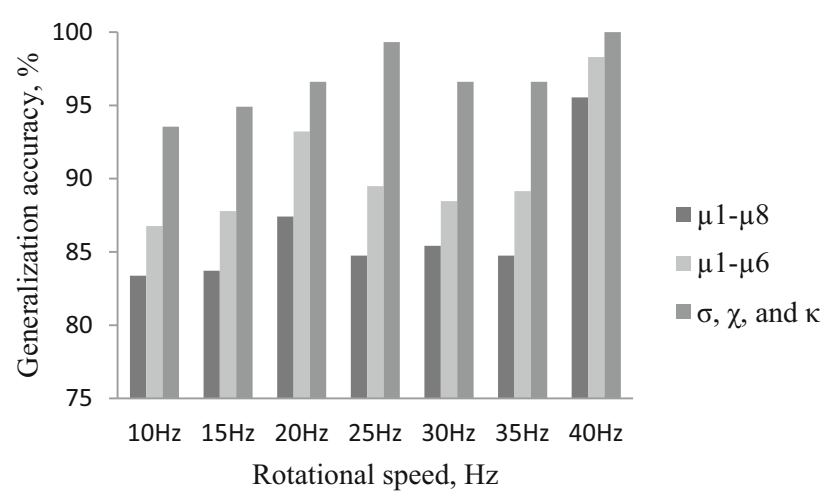

Fig. 8 A parametric study on the selection of optimum statistical features

\section{Selection of Optimum Statistical Features for Fault Diagnosis of IMs}

The effect of different statistical features on the generalization accuracy has been examined and selected the best statistical features for further fault classification. Initially, eight higher statistical moments are extracted from raw time domain vibration signals in $x, y$, and $z$-directions for the highest torque condition. The overall generalization accuracies are compared among all eight higher statistical moments, six higher statistical moments, and three statistical moments (standard deviation- $\sigma$, skewness- $\chi$, and kurtosis- $\kappa$ ) as shown in Fig. 8. For this exercise, the training and testing data are selected arbitrarily in the ratio of 4:1 and number of datasets as 300 . Later the optimum value of these parameters will also be checked, i.e. the optimum ratio of training and testing datasets. Result shows that three statistical features $(\sigma, \chi$, and $\kappa)$ gives the highest generalization accuracy at all rotational speeds as compare to eight and six higher statistical moments, and are shown in Table 1. Correspondingly, the best fixed $\gamma$ value used for analysis is also presented. Hence, now these three statistical features will be used as optimum features for further exercise of the fault diagnosis.

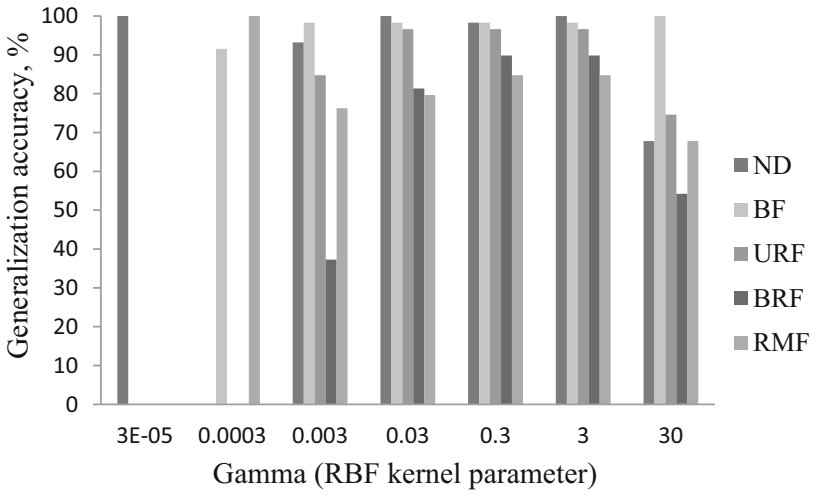

Fig. 9 A parametric study on the selection of optimum value of $\gamma$ at $10 \mathrm{~Hz}$ rotational speed

\section{Selection of the Optimum RBF Kernel Parameter (Gamma- $\gamma$ ) for the Fault Diagnosis of IMs}

The effect of different $\gamma$ values on the generalization accuracy has been examined and selected the best value of $\gamma$ for all further fault classifications. For this exercise optimum three statistical features $(\sigma, \chi$, and $\kappa)$ have been chosen and arbitrarily selected the training and testing data in the ratio of $4: 1$, total number of datasets as 300 and rotational speeds of 10 and $40 \mathrm{~Hz}$ for the highest torque. The generalization accuracy by using the SVM for 10 and $40 \mathrm{~Hz}$ speeds are shown in Figs. 9 and 10, respectively. The maximum generalization accuracy is obtained at the $\gamma$ value of 0.3 for the higher as well as lower speeds. So now $\gamma=0.3$ has been used as the optimum value for further fault diagnosis exercises.

\section{Selection of the Optimum Training and Testing Data Percentage for the Fault Diagnosis of IMs}

The effect of different training and testing data percentage have been examined on the generalization accuracy and selected the best percentage of training and testing data for further fault classifications. For this exercise, optimum three

Table 1 Selection of optimum statistical features

\begin{tabular}{|c|c|c|c|c|}
\hline \multirow[t]{2}{*}{ S. no. } & \multirow[t]{2}{*}{ Training-testing speeds (HZ) } & \multicolumn{3}{|c|}{ Maximum overall generalization accuracy/respective $\gamma$} \\
\hline & & Statistical features $\left(\mu_{1}-\mu_{8}\right)$ & Statistical features $\left(\mu_{1}-\mu_{6}\right)$ & $\begin{array}{l}\text { Statistical features } \\
(\sigma, \chi, \text { and } \kappa)\end{array}$ \\
\hline 1 & 40 & $95.55 / 3 \times 10^{-18}$ & $98.30 / 3 \times 10^{-13}$ & $100 / 3 \times 10^{-1}$ \\
\hline 2 & 35 & $84.75 / 3 \times 10^{-16}$ & $89.15 / 3 \times 10^{-11}$ & $96.61 / 3 \times 10^{-1}$ \\
\hline 3 & 30 & $85.42 / 3 \times 10^{-16}$ & $88.47 / 3 \times 10^{-11}$ & $96.61 / 3 \times 10^{-1}$ \\
\hline 4 & 25 & $84.75 / 3 \times 10^{-15}$ & $89.49 / 3 \times 10^{-10}$ & $99.32 / 3 \times 10^{-1}$ \\
\hline 5 & 20 & $87.42 / 3 \times 10^{-13}$ & $93.22 / 3 \times 10^{-9}$ & $96.61 / 3 \times 10^{-1}$ \\
\hline 6 & 15 & $83.72 / 3 \times 10^{-11}$ & $87.79 / 3 \times 10^{-8}$ & $94.91 / 3 \times 10^{-1}$ \\
\hline 7 & 10 & $83.38 / 3 \times 10^{-8}$ & $86.77 / 3 \times 10^{-6}$ & $93.55 / 3 \times 10^{-1}$ \\
\hline
\end{tabular}

Bold values represent the best generalization accuracy of considered cases 


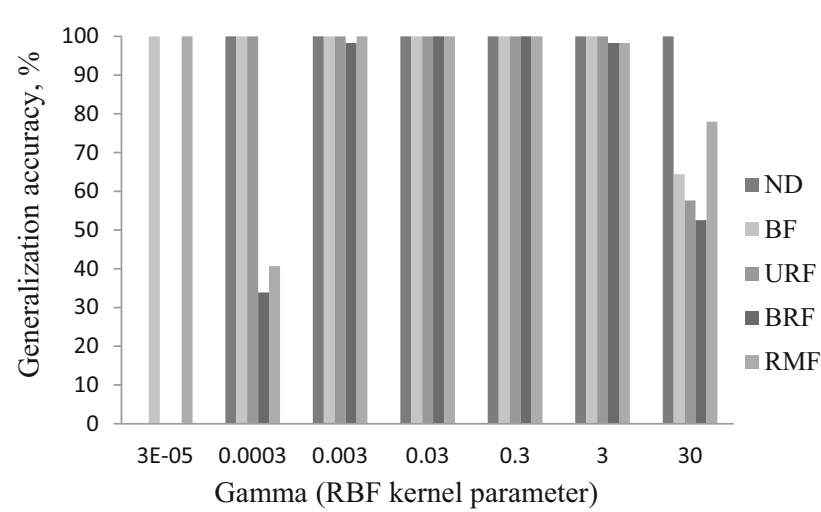

Fig. 10 A parametric study on the selection of optimum value of $\gamma$ at $40 \mathrm{~Hz}$ rotational speed

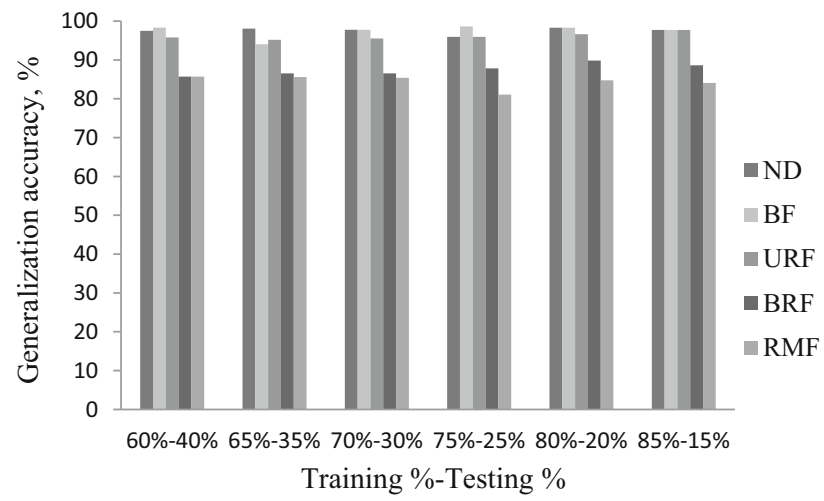

Fig. 11 A parametric study on the selection of optimum training and testing data percentage at $10 \mathrm{~Hz}$ speed

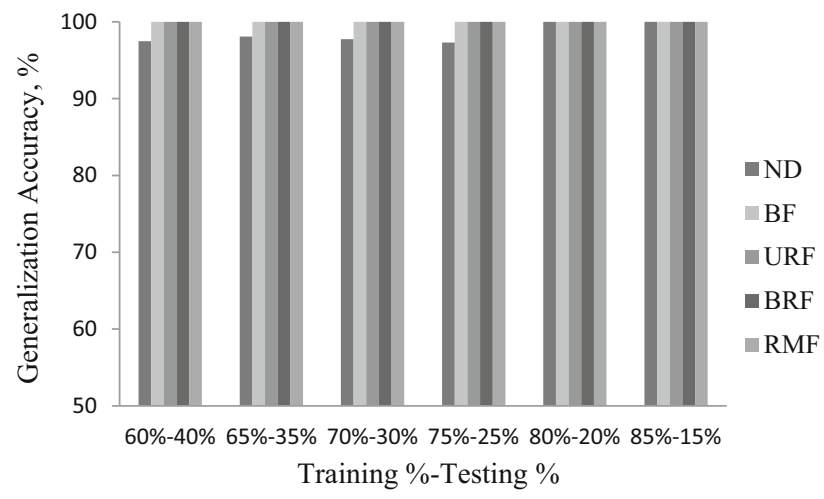

Fig. 12 A parametric study on the selection of optimum training and testing data percentage at $40 \mathrm{~Hz}$ speed

statistical features $(\sigma, \chi$ and $\kappa)$, the optimum $\gamma=0.3$, arbitrarily selected number of datasets as 300 and rotational speeds of 10 and $40 \mathrm{~Hz}$ for the highest torque have been chosen. The generalization accuracy by using the SVM for 10 and $40 \mathrm{~Hz}$ speeds are shown in Figs. 11 and 12, respectively. The maximum generalization accuracy appeared at the ratio of $80-20 \%$ of training and testing data, respectively, for the higher as well as lower speeds. Often accuracy increases with training percentages, however Fig. 11 shows that accuracy decreases somewhat when training percentage increases from 80 to $85 \%$. Hence, now $80-20 \%$ of the training and testing data, respectively, will be used as an optimum percentage for further fault diagnosis exercises.

\section{Selection of the Optimum Number of Feature Datasets for the Fault Diagnosis of IMs}

This particular exercise has been done to select minimum number of feature datasets for the perfect classification through SVM. It is known that accuracy increases with feature datasets. However, it cannot consider large number of datasets arbitrarily because it can cause computational burden. For this exercise, optimum three statistical features ( $\sigma, \chi$ and $\kappa$ ), the optimum $\gamma$ value of 0.3 and the optimum training-testing data percentage of $80-20 \%$ have been chosen, and checked for different number of feature datasets at 10 and $40 \mathrm{~Hz}$ rotational speeds for the highest torque. The generalization accuracy by using the SVM for 10 and $40 \mathrm{~Hz}$ speeds are shown in Figs. 13 and 14, respectively. The maximum generalization accuracy appears at the value of 300

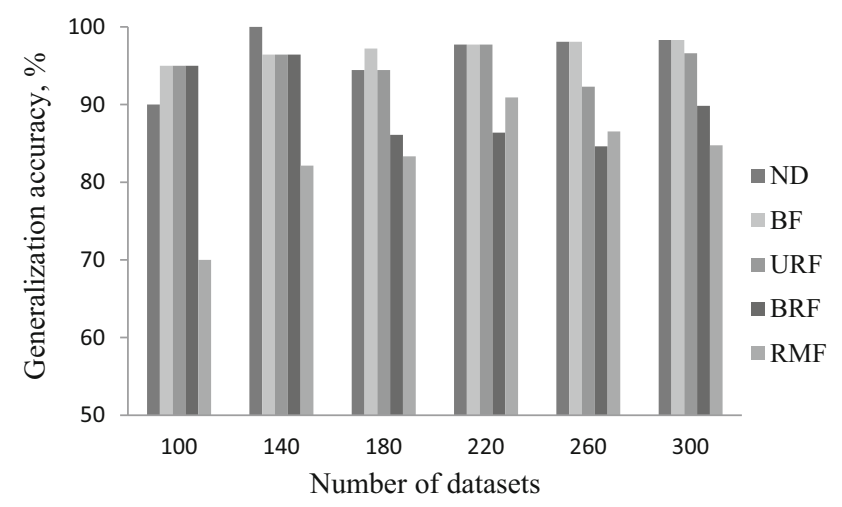

Fig. 13 A parametric study of the selection of optimum number of feature datasets at $10 \mathrm{~Hz}$ speed

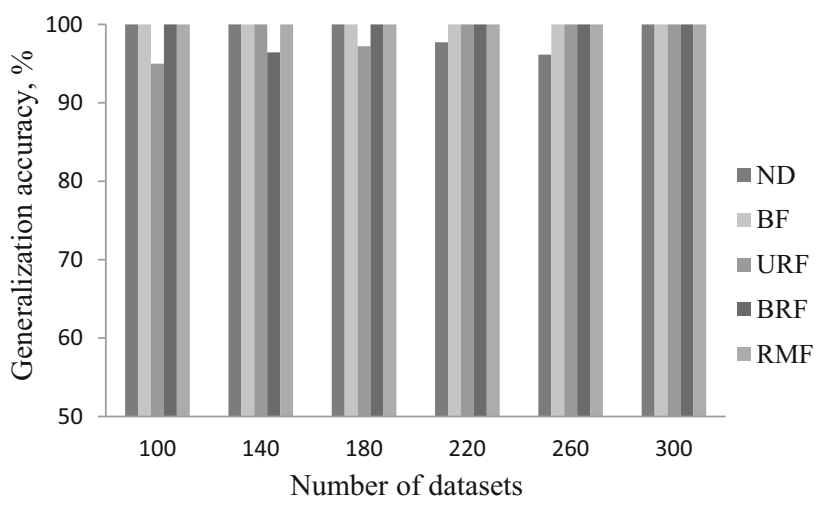

Fig. 14 A parametric study of the selection of optimum number of feature datasets at $40 \mathrm{~Hz}$ speed 
Table 2 Classification of IM faults with the training and the testing at same rotational speed condition

\begin{tabular}{|c|c|c|c|c|c|c|c|}
\hline \multirow[t]{2}{*}{ Training speed $(\mathrm{Hz})$} & \multirow{2}{*}{$\begin{array}{l}\text { Testing speed } \\
(\mathrm{Hz})\end{array}$} & \multicolumn{6}{|c|}{ Generalization accuracy, $\%$} \\
\hline & & ND & $\mathrm{BF}$ & URF & $\mathrm{BRF}$ & $\mathrm{RMF}$ & $\begin{array}{l}\text { Overall } \\
\text { accuracy }\end{array}$ \\
\hline \multicolumn{8}{|l|}{ a. For lowest torque } \\
\hline 10 & 10 & 79.66 & 100 & 94.92 & 81.36 & 66.10 & 84.40 \\
\hline 15 & 15 & 88.14 & 100 & 100 & 84.75 & 93.22 & 93.22 \\
\hline 20 & 20 & 96.61 & 100 & 98.31 & 96.61 & 100 & 98.30 \\
\hline 25 & 25 & 93.22 & 100 & 83.05 & 93.22 & 98.31 & 93.55 \\
\hline 30 & 30 & 94.92 & 100 & 79.66 & 91.53 & 96.61 & 92.54 \\
\hline 35 & 35 & 94.92 & 100 & 94.92 & 100 & 94.92 & 96.94 \\
\hline 40 & 40 & 93.22 & 100 & 94.92 & 98.31 & 98.31 & 96.94 \\
\hline \multicolumn{8}{|c|}{ b. For medium torque } \\
\hline 10 & 10 & 86.44 & 100 & 93.22 & 81.36 & 86.44 & 89.83 \\
\hline 15 & 15 & 77.92 & 100 & 96.61 & 89.83 & 93.22 & 91.52 \\
\hline 20 & 20 & 94.92 & 100 & 100 & 96.61 & 100 & 98.30 \\
\hline 25 & 25 & 98.31 & 100 & 100 & 98.31 & 100 & 99.32 \\
\hline 30 & 30 & 98.31 & 100 & 67.80 & 98.31 & 100 & 92.88 \\
\hline 35 & 35 & 94.92 & 100 & 96.61 & 94.92 & 100 & 97.28 \\
\hline 40 & 40 & 100 & 100 & 94.92 & 100 & 100 & 98.98 \\
\hline \multicolumn{8}{|l|}{ c. For highest torque } \\
\hline 10 & 10 & 98.31 & 98.31 & 96.61 & 89.83 & 84.75 & 98.31 \\
\hline 15 & 15 & 86.44 & 100 & 98.31 & 96.61 & 93.22 & 94.91 \\
\hline 20 & 20 & 86.44 & 100 & 98.31 & 98.31 & 100 & 96.61 \\
\hline 25 & 25 & 100 & 100 & 98.31 & 98.31 & 100 & 99.32 \\
\hline 30 & 30 & 93.22 & 100 & 94.92 & 96.61 & 98.31 & 96.61 \\
\hline 35 & 35 & 94.92 & 100 & 89.83 & 98.31 & 100 & 96.61 \\
\hline 40 & 40 & 100 & 100 & 100 & 100 & 100 & 100 \\
\hline
\end{tabular}

Bold values represent the best individual and overall generalization accuracy in considered cases feature datasets for the higher as well as lower speeds that means minimum 300 datasets are required for perfect SVM classification. Hence, now 300 feature datasets will be used as an optimum percentage for further fault diagnosis exercises.

\section{Fault Diagnosis with the Testing at the Same Rotational Speeds as the Training}

In this case, the SVM classifier is trained and tested with the optimum number of time domain statistical features at the same rotational speed. Rotational speeds selected are $10-40 \mathrm{~Hz}$ in a step of $5 \mathrm{~Hz}$. To demonstrate the proposed fault diagnosis of IMs based on the multiclass SVM, the following parameters have been used: three optimum statistical features $(\sigma, \chi$ and $\kappa)$, the optimum $\gamma$ value as 0.3 , the optimum training-testing data percentage as $80-20 \%$, and the optimum number of feature datasets as 300 . By using these optimal parameters a SVM model based on one-versus-one approach and Gaussian RBF kernel is build and trained which will be used for the final fault diagnosis of IMs. The num- ber of experiment and data generation have been performed by varying the load on induction motor, i.e. (1) the lowest torque or no load- $0 \mathrm{~N}-\mathrm{m}$, (2) the medium torque or light load $-0.113 \mathrm{~N}-\mathrm{m}$ and (3) the highest torque or full load$0.565 \mathrm{~N}-\mathrm{m}$. Finally the multiclass fault diagnosis of IMs has been performed by using optimal parameters found in parametric studies and the final results of the fault classification are obtained in the percentage of generalization accuracy. Table 2 shows that the mechanical fault classification of IMs for different rotational speed at the lowest, medium and highest torques.

\section{Fault Classification Ability}

The result shows that, the generalization accuracy (in \%), i.e. the percentage of successfully predicted data, by using the SVM for three values of torques. The maximum value of overall (average) generalization accuracy came $98.30 \%$ at $20 \mathrm{~Hz}, 99.32 \%$ at $25 \mathrm{~Hz}$ and $100 \%$ at $40 \mathrm{~Hz}$ rotational speed, and the minimum accuracy came $84.40 \%$ at $10 \mathrm{~Hz}, 89.83 \%$ at $10 \mathrm{~Hz}$ and $93.55 \%$ at $10 \mathrm{~Hz}$ for the lowest, medium and 
Table 3 Classification of IM faults with the training and the testing at the interpolation speed condition (range 10 Hz)

\begin{tabular}{|c|c|c|c|c|c|c|c|}
\hline \multirow[t]{2}{*}{ Training speed $(\mathrm{Hz})$} & \multirow[t]{2}{*}{ Testing speed $(\mathrm{Hz})$} & \multicolumn{6}{|c|}{ Generalization accuracy, $\%$} \\
\hline & & ND & $\mathrm{BF}$ & URF & BRF & $\mathrm{RMF}$ & Overall accuracy \\
\hline \multicolumn{8}{|l|}{ a. For lowest torque } \\
\hline 10,20 & 15 & 62.71 & 100 & 86.44 & 74.58 & 59.32 & 76.61 \\
\hline 15,25 & 20 & 83.05 & 100 & 94.92 & 96.61 & 100 & 94.91 \\
\hline 20,30 & 25 & 45.76 & 100 & 96.61 & 72.88 & 100 & 83.05 \\
\hline 25,35 & 30 & 91.53 & 100 & 79.66 & 98.31 & 76.27 & 89.15 \\
\hline 30,40 & 35 & 83.05 & 100 & 89.83 & 94.92 & 96.61 & 92.88 \\
\hline \multicolumn{8}{|l|}{ b. For medium torque } \\
\hline 10,20 & 15 & 61.02 & 100 & 84.75 & 76.27 & 74.58 & 79.32 \\
\hline 15,25 & 20 & 62.71 & 100 & 100 & 100 & 96.61 & 91.86 \\
\hline 20,30 & 25 & 100 & 100 & 94.92 & 100 & 100 & 98.98 \\
\hline 25,35 & 30 & 96.61 & 100 & 55.93 & 100 & 100 & 90.5 \\
\hline 30,40 & 35 & 84.75 & 88.14 & 98.31 & 96.61 & 100 & 93.55 \\
\hline \multicolumn{8}{|l|}{ c. For highest torque } \\
\hline 10,20 & 15 & 86.44 & 64.41 & 100 & 76.27 & 83.05 & 82.03 \\
\hline 15,25 & 20 & 71.19 & 100 & 100 & 98.31 & 94.92 & 92.88 \\
\hline 20,30 & 25 & 98.31 & 100 & 93.22 & 100 & 100 & 98.3 \\
\hline 25,35 & 30 & 96.61 & 100 & 91.53 & 91.53 & 96.61 & 95.25 \\
\hline 30,40 & 35 & 81.36 & 100 & 94.92 & 100 & 98.31 & 94.91 \\
\hline
\end{tabular}

Bold values represent the best individual and overall generalization accuracy in considered cases

highest torques, respectively. That shows the average generalization accuracy and the rotational speed with respect to the maximum value of overall generalization accuracy increases with the torque applied on IMs. This is because the whole system (experiment setup) became more stiff (hence signal-to-noise ratio enhanced) with respect to the increase in the torque. And with the increase in the rotational speed the fault dynamics becomes predominant leading again to a better signal-to-noise ratio.

The individual generalization accuracy for each fault condition (i.e. BF, URF, BRF, RMF and ND) is also shown in Table 2. For the lowest torque, the worst individual generalization accuracy obtained for RMF of $66.10 \%$ at lowest speed of $10 \mathrm{~Hz}$ and the highest accuracy obtained of $100 \%$ for $\mathrm{BF}$ at all speeds, for URF at $15 \mathrm{~Hz}$, for BRF at $35 \mathrm{~Hz}$ and for RMF at $20 \mathrm{~Hz}$. For the medium torque value, it came the worst accuracy for URF of $67.80 \%$ at $30 \mathrm{~Hz}$ speed and the highest accuracy of $100 \%$ for $\mathrm{BF}$ at all speeds, for RMF at all speeds except 10 and $15 \mathrm{~Hz}$, for URF at 20 and $25 \mathrm{~Hz}$, and for ND and RMF at $40 \mathrm{~Hz}$. For the highest value of torque, it came the worst accuracy for RMF of $84.75 \%$ at $10 \mathrm{~Hz}$ and the highest accuracy of $100 \%$ for BF at all speeds except $10 \mathrm{~Hz}$, for RMF at $20,25,35$ and $40 \mathrm{~Hz}$, for ND at 25 and $40 \mathrm{~Hz}$, and for URF and BRF at $40 \mathrm{~Hz}$. Hence, the result shows the individual generalization accuracy for each fault condition increases with the rotational speed as well as loading (torque) for the reason mentioned above.
It also to be noted that for the individual fault classification the individual generalization accuracy in minimummaximum range comes nearly $98.30-100 \%$ for IM with bearing fault (BF), $79.66-100 \%$ for ND, $67.80-100 \%$ for URF, $81-100 \%$ for BRF and $66.10-100 \%$ for RMF at all rotational speeds and for all torques. That means BF can be perfectly classified for any load at any speed by the SVM based on time domain vibration signals. While for other mechanical fault conditions the SVM is predicting nearly perfect at higher speeds and mainly at higher torques.

\section{Fault Diagnosis at an Intermediate Rotational Speed for Which the SVM was not Trained}

In this case, the SVM classifier is trained at two rotational speeds and tested at an intermediate rotational speed for which the classifier was not trained. Table 3 shows the value of training speed ranges and intermediate testing rotational speeds. Here the speed range, $10 \mathrm{~Hz}$ is considered, because beyond this range it would be too wide hence generalization accuracy becomes low. To demonstrate the proposed fault diagnosis of IMs based on the multiclass SVM, the following parameters have been used: three optimum statistical features $(\sigma, \chi$ and $\kappa)$, the optimum training-testing data percentage as $80-20 \%$, and the optimum number of feature datasets as 300. For this case, the optimum value of $\gamma$ is 0.03 unlike the previous case, i.e. training and testing at the same rotational speed. By using these optimal parameters a 
SVM model based on the one-versus-one approach and the Gaussian RBF kernel is build and trained which is used for the final fault diagnosis of IMs.

\section{Fault classification ability}

Result shows that the generalization accuracy, by using the SVM for three values of torque, at the intermediate rotational speed ranges of $10 \mathrm{~Hz}$. The maximum value of overall (average) generalization accuracy came $94.91 \%$ at $20 \mathrm{~Hz}, 99.98 \%$ at $25 \mathrm{~Hz}$ and $98.3 \%$ at $20 \mathrm{~Hz}$ interpolated rotational speed, and minimum came $76.61 \%$ at $15 \mathrm{~Hz}, 79.32 \%$ at $15 \mathrm{~Hz}$ and $82.03 \%$ at $15 \mathrm{~Hz}$ for the lowest, medium and highest torques, respectively. That shows the average generalization accuracy and the interpolated rotational speed with respect to the maximum value of overall generalization accuracy increases somewhat with the torque applied on IMs.

The individual generalization accuracy for each faulty condition (i.e. BF, URF, BRF, RMF and ND) is also shown in Table 3. For the lowest torque, the worst individual generalization accuracy obtained for ND of $45.76 \%$ at interpolated speed of $25 \mathrm{~Hz}$ and the highest accuracy obtained of $100 \%$ for $\mathrm{BF}$ at all interpolated speeds, for RMF at 20 and $25 \mathrm{~Hz}$. For the medium torque value it came worst accuracy for URF of $55.93 \%$ at $30 \mathrm{~Hz}$ interpolated speed and highest accuracy of $100 \%$ for $\mathrm{BF}$ at all interpolated speeds except $35 \mathrm{~Hz}$, for ND at $25 \mathrm{~Hz}$, for URF at $20 \mathrm{~Hz}$, for BRF at 20,25 and $30 \mathrm{~Hz}$, and for RMF at all speeds except 15 and $20 \mathrm{~Hz}$. For the highest value of torque it came worst accuracy for BF of $64.41 \%$ at $15 \mathrm{~Hz}$ and the highest accuracy of $100 \%$ for $\mathrm{BF}$ at all speeds except $15 \mathrm{~Hz}$, for RMF at $25 \mathrm{~Hz}, \mathrm{BRF}$ at 25 and $35 \mathrm{~Hz}$, and for URF at 15 and $20 \mathrm{~Hz}$. Hence, here also the result shows the individual generalization accuracy for each fault condition increases with the rotational speed as well as loading (torque). Also it is observed that the BF can be perfectly classified for any load at any interpolated speeds similar like same rotational speed conditions.

For the classification of response data in time domain for the linear system model, the SVM and ANN algorithms use a similar concept of the pattern recognition. However, for the non-linear system, they use different classification methodology. To deal with the non-linearity, the ANN utilizes various activation functions and multilayer connections. However, the SVM employs a kernel function to deal with the nonlinearity by mapping the non-linear data in a low dimensional space to a high dimensional space. The ANN are based on the empirical risk minimization (ERM) as it minimizes the error on training data only. On the other hand, the SVM are based on structural risk minimization (SRM) because it minimizes the upper bound on expected risk. The SRM is superior to the ERM in terms of generalization ability.

For the estimation of induction motor parameters such as rotor current and speed, and the stator voltage and current, the Kalman filter algorithm have also been widely used. By using extended Kalman filter algorithm, the estimation of states and parameters can be performed simultaneously of the non-linear system. This algorithm requires terminal and rotor speed measurement. This algorithm mainly used for parameters and state estimation, however, finally for the fault diagnosis of induction motor the ANN and recently the SVM algorithm is more popular.

For the diagnosis of induction motor faults, condition monitoring techniques have been developed based on frequency domain analysis such as the FFT, power spectrum, bispectrum and high resolution spectral analysis. The spectrum analysis of vibration signals have been used to distinguish the mechanical faults. The first harmonic of vibration force may arise in the spectrum for a number of faulty states such as the rotor unbalance, bowed shaft and rotor misalignment. The bowed and misaligned rotor can be separated from unbalanced rotor if a large vibration component occurs at second harmonics. Note that this component does not occur for the unbalanced rotor. By identifying the pre-dominant vibration plane, i.e. radial or axial and the relative phase between two ends of shaft, the bowed rotor can be separated from the parallel as well as angular misaligned rotor. The bearing fault can be identified in the vibration spectrum by carefully observing various fault frequencies. Thomson and Orpin [28] used spectrum analysis of the current and vibration signals for the fault diagnosis of induction motors. The extensive analyses of behavior of sidebands associates with different faults were analyzed. For consistent fault diagnosis, tracking of the accurate narrow-bands and sidebands in a spectrum is essential and it depends on the frequency resolution.

The frequency information can be extracted using FFT but it is not possible to mark at what instances these frequency components occur. For non-stationary signals (i.e., spectral content vary with the time), wavelets such as continuous wavelet transform, Hilbert-Hung transform and WignerVille distribution, are preferred [29]. The windowed Fourier transform (WFT), a robust and simple time-frequency method has also been widely used for induction motor fault diagnosis. Nowadays the wavelet transform is used for fault diagnosis in conjunction with the SVM.

The competence of these time, frequency and timefrequency methods can be characterized by their capability to diagnose the faulty conditions in motors. To distinguish faulty conditions from normal state, based on the spectral analysis, it is required to have adequate knowledge and expertise. Moreover, it requires manual inspection of signal characteristics. The FFT contains a large number of spectral content, i.e. harmonics and sub-harmonics of fundamental components, however by using expert systems such as the SVM (or ANN), undesirable harmonic information can be filtered out and the suitable one for effective fault diagnosis may be used. Connectionist networks such as SVM used for 
the fault classification purposes can be fed by a data base which contains the machine history in terms of trends of well-stated failure components. A good fault classifier will be capable of recognizing these failure components correctly, to classify appropriate failure modes. Moreover, if a suitable normalization is performed on input feature sets, then the trained SVM can be applied to a family of similar induction motors.

\section{Conclusions}

In the present work, the multiclass classification of mechanical faults in IMs based on time domain vibration signature by using the one-versus-one multiclass approach of the SVM has been applied. Initially eight higher statistical moments were extracted as features from time domain vibration signals and finally found that only three optimum statistical features $(\sigma, \chi$ and $\kappa)$ are effective. Also the optimum $\gamma$ value, optimum training-testing data ratio and optimum number of feature datasets are chosen through parametric studies. Then, the SVM capability to classify mechanical faults in IMs has been presented when training and testing of SVM is done at the same rotational speed as measured data, and innovatively when the SVM is tested at an intermediate rotational speed for which classifier was not trained. The prediction accuracy of classifying faults show near perfect prediction performance when prediction attempt at the same rotational speed as measured data condition. And it shows relatively better prediction performance when prediction attempt also at the interpolated rotational speed condition. It also shows that the average generalization accuracy increases with the rotational speeds as well as the torque applied on IMs, It is also observed that the individual generalization accuracy for each fault condition increases with rotational speeds as well as loadings. A perfect prediction has been found for the BF for any load at any speed considered, while for other mechanical fault conditions the SVM is predicting nearly perfect at higher speeds and mainly for higher torques. The present methodology could be applied for the training and testing of SVM based on frequency and time-frequency domain data also.

Acknowledgments We would like to thank Dr. D. J. Bordoloi and Mr. Sandeep Singh, Vibration and Acoustic Laboratory, Department of Mechanical Engineering, IIT Guwahati, for their useful help during the experimentation. This work is supported by LIBSVM tool, which is freely available online at-http://www.csie.ntu.edu.tw/ cjlin/libsvm.

\section{References}

1. Siyambalapitiya, D.J.T., McLaren, P.G.: Reliability improvement and economic benefits of on-line monitoring system for large induction machines. IEEE Trans. Ind. Appl. 26(6), 1018-1025 (1990)
2. Zhongming, Y., Bin, W.: A review on induction motor online fault diagnosis. Proceedings of the IEEE Third International Conference on Power Electronics and Motion Control, IPEMC 3, 1353-1358 (2000)

3. Timusk, M., Lipsett, M., Mechefske, C.K.: Fault detection using transient machine signals. Mech. Syst. Signal Process. 22(7), 17241749 (2008)

4. Kral, C., Habetler, T.G., Harley, R.G., Pirker, F., Pascoli, G., Oberguggenberger, H., Fenz, C.J.M.: A comparison of rotor fault detection techniques with respect to the assessment of fault severity. In: Proceedings of the 4th IEEE International Symposium on Diagnostics for Electric Machines, Power Electronics and Drives, SDEMPED, pp. 265-270 (2003)

5. Nguyen, N.T., Lee, H.H.: An application of support vector machines for induction motor fault diagnosis with using genetic algorithm. In: Huang, D.-S., Wunsch II, D.-C., Levine, D.-S., Jo, K.-H. (eds.)International Conference on Intelligent Computing (ICIC-2008), pp. 190-200. Springer, Heidelberg (2008)

6. Tran, V.T., Yang, B.S., Oh, M.S., Tan, A.C.C.: Fault diagnosis of induction motor based on decision trees and adaptive neuro-fuzzy inference. Expert Syst. Appl. 36(2), 1840-1849 (2009)

7. Park, W.J., Lee, S.H., Joo, W.K., Song, J.I.: A mixed algorithm of PCA and LDA for fault diagnosis of induction motor. In: Huang, D.-S., Heutte, L., Loog, M. (eds.) International Conference on Intelligent Computing (ICIC-2007), pp. 934-942. Springer, Heidelberg (2007)

8. Baccarini, L.M.R.: Rocha e Silva, V.V., De Menezes, B.R., Caminhas, W.M.: SVM practical industrial application for mechanical faults diagnostic. Expert Syst. Appl. 38(6), 6980-6984 (2011)

9. Samanta, B.: Gear fault detection using artificial neural networks and support vector machines with genetic algorithms. Mech. Syst. Signal Process. 18(3), 625-644 (2004)

10. Silva, V.A.D., Pederiva, R.: Fault detection in induction motors based on artificial intelligence. In: Proceedings of the International Conference on Surveillance 7. Institute of Technology of Chartres, France (2013)

11. Hsu, C.W., Lin, C.J.: A comparison of methods for multiclass support vector machines. IEEE Trans. Neural Netw. 13(2), 415-425 (2002)

12. Hsu, C.W., Chang, C.C., Lin, C.J.: A practical guide to support vector classification. http://www.csie.ntu.edu.tw/ cjlin/papers/guide/ guide.Pdf (2003)

13. Li, X., Zheng, A., Zhang, X., Li, C., Zhang, L.: Rolling element bearing fault detection using support vector machine with improved ant colony optimization. Measurement 46, 2726-2734 (2013)

14. Tiwari, R., Bordoloi, D.J.: Optimization of support vector machine based multi-fault classification with evolutionary algorithms from time domain vibration data of gears. Proc. Inst. Mech. Eng. Part C J. Mech. Eng. Sci. 227(11), 2428-2439 (2013)

15. Widodo, A., Yang, B.S., Han, T.: Combination of independent component analysis and support vector machines for intelligent faults diagnosis of induction motors. Expert Syst. Appl. 32(2), 299-312 (2007)

16. Martínez-Morales J.D., Palacios, E., Campos-Delgado, D.U.: Data fusion for multiple mechanical fault diagnosis in induction motors at variable operating conditions. In: Proceedings of the IEEE 7th International Conference on Electrical Engineering Computing Science and Automatic Control (CCE), pp. 176-181 (2010)

17. Chattopadhyay, P., Konar, P.: Feature extraction using wavelet transform for multi-class fault detection of induction motor. J. Inst. Eng. (India) Ser. B 95(1), 73-81 (2014)

18. Uddin, J., Kang, M., Nguyen, D.V., Kim, J.M.: Reliable fault classification of induction motors using texture feature extraction and a multiclass support vector machine. Mathematical Problems in Engineering, Hindawi Corp., vol. 2014, pp. 1-9 (2014) 
19. Shahriar, M.R., Ahsan, T., Chong, U.: Fault diagnosis of induction motors utilizing local binary pattern-based texture analysis. EURASIP J. Image Video Process. 2013(1), 1-11 (2013)

20. Vapnik, V., Levin, E., Le, C.Y.: Measuring the VC-dimension of a learning machine. Neural Comput. 6(5), 851-876 (1994)

21. Vapnik, V.N.: An overview of statistical learning theory. IEEE Trans. Neural Netw. 10(5), 988-999 (1999)

22. Burges, C.J.: A tutorial on support vector machines for pattern recognition. Data Min. Knowl. Discov. 2(2), 121-167 (1998)

23. Gunn, S.R.: Support vector machines for classification and regression. Department of Electronics and Computer Science of University of Southampton, Southampton (1998)

24. Boser, B.E., Guyon, I.M., Vapnik, V.N.: A training algorithm for optimal margin classifiers. In: Proceedings of the Fifth Annual Workshop on Computational Learning Theory, pp. 144-152. ACM (1992)

25. Chang, C.C., Lin, C.J.: LIBSVM: a library for support vector machines. ACM Trans. Intell. Syst. Technol. 2(3), 27 (2011)
26. Toyota, T., Niho, T., Chen, P.: Failure detection and diagnosis of rotating machinery by orthogonal expansion of density function of vibration signal. In: Proceedings of the IEEE EcoDesign'99: First International Symposium on Environmentally Conscious Design and Inverse Manufacturing, pp. 886-891 (1999)

27. Zhang, L., Jack, L.B., Nandi, A.K.: Fault detection using genetic programming. Mech. Syst. Signal Process. 19(2), 271-289 (2005)

28. Thomson, W.T., Orpin, P.: Current and vibration monitoring for fault diagnosis and root cause analysis of induction motor drives. In: Proceedings of the thirty-first turbomachinery symposium, pp. 61-67 (2002)

29. Rosero, G., Martínez, J.A., R., J. L., Rosero, E., Betancourt, U., César, J.: Fault detection in dynamic conditions by means of discrete wavelet decomposition for PMSM running under bearing damage. In: Twenty-Fourth Annual IEEE Applied Power Electronics Conference and Exposition, pp. 951-956. Institute of Electrical and Electronics Engineers, IEEE Press (2009) 